\title{
Factors influencing the dispersion of Arceuthobium oxycedri in Central Spain: evaluation with a new null model for marked point patterns
}

\author{
By P. Ramón ， M. De la Cruz , I. Zavala and M. A. Zavala
}

\begin{abstract}
Summary
The dwarf mistletoe, Arceuthobium oxycedri, is found on populations of jumiperus oxycedrus, in central Spain. This species can lave negative effects on the physiology of its host, including mortality. Understanding the mechanisms that control its distribution and dispersal is critical to assessing its potential for spread. We assessed dwarf mistletoe distribution within a population of $l$, oxycedrus, including infected and uninfected host individuals. A new null model of parasitic dispersion was built using two dispersal kernel forms that were simulated with lower and upper envelopes for second-order functions to summarize a point pattern, sucls as Ripley's $K$, nearest-neighbour distribution and pair correlation functions. Nine dispersal scenarios were constructed with half-bandwidth kernels $(10,20,30 \mathrm{~m})$ and initial population of infected trees $\left(P_{0}=05,10\right.$ and 20). These scenarios were compared with the observed pattern and evaluated using the goodnessof-fit test. Significant differences at short distance $(r<10 \mathrm{~m})$ were found between the observed pattern and simulated patterns, corresponding to the range of seed dispersal of the dwarf mistletoe. Interactions between infected and uninfected hosts patterns at all scales were identified, suggesting that $\boldsymbol{A}$. oxycedri uses other mechanisms in addition to ballistic seed shooting as secondary dispersal agents to spread to distances greater than $20 \mathrm{~m}$. Given that the seed characteristics facilitate dispersal by adhesion, we infer that spread between host individuals is amplified by seed transport by birds or small mammals.
\end{abstract}

\section{Introduction}

Many types of mistletoes are both mutualistic with animal vectors, and parasitic on host plants (Aukema 2003). However, the explosively dispersed dwarf mistletoes (Arceuthobium spp.) and wind-dispersed Misodendraceae have no mutualistic animal dispersers. Mistletoe fruits have adhesive seeds that stick by viscous excretions to a host branch. In plants, dispersal vectors can act at different scales and generate different dispersal kernels for a particular species (Nathan 2007). Mistletoe dispersal is generally localized around the host plant (Maloney and Rizzo 2002) and it is considered random (Overton 1994). However, some studies suggest a non-random mistletoe distribution among host plants (Larson 1996; Wenny 2001). Birds carrying seeds to already infected hosts may increase seed rain within the patches already infected, resulting in a preferential dispersal (Aukema and Martinez del Río 2002). Mistletoe's dispersal form is a complex process that involves habitat factors as well as animal behaviour, and it is influenced by woodland fragmentation (Lavorel et al. 1999).

Arceuthobium oxycedri DC. M. Bieb is the most widely distributed species of Arceuthobium, reported in 31 countries from Spain to western China (Ciesla et al. 2004). It parasitizes native and introduced species of Juniperus particularly Juniperus oxycedrus (L.) and Juniperus communis, and several other introduced species in the Cupressaceae family (Ríos Insua 1987; Ciesla et al. 2004). Fruiting and seed dispersal occurs between October and November, with a maturity period of 13 months (Catalán 1997). Dispersal is provided primarily by the ballistic mechanism of the plant itself (Catalán 1997), but birds may be indirect dispersal vectors if seeds adhere to the bird and are then transferred to other hosts (Zilka and Tinnin 1976; Ostry et al. 1983). Birds have been suggested as long-distance vectors of A. oxycedri in France and Pakistan, because song thrush ingests fruits of mistletoe, but quantitative data are lacking (Hawksworth and Geils 1996). Arceuthobium oxycedri impacts its host through reduction in seed production and wood quality, and by making hosts more susceptible to fungal attack (Ríos Insua 1987); however, the mistletoes can provide habitat and a food source for some species of birds, insects and small mammals (Maloney and Rizzo 2002). This species of mistletoe is also known for its medicinal properties (Akkol et al. 2010).

Hypothetically, the mode of seed dispersal of A. oxycedri is autochory with epizoochory, that is characterized by self-dispersal (physical and often explosive discharge of seeds from the fruit). The secondary transport or directed dispersal occurs when seed attached to the bird's body are transported (Watson and Rawsthorne 2013). Previous studies have identified, in certain species of birds, that they carry Arceuthobium seeds attached to their feathers (Nicholls et al. 1984). Juniperus oxycedrus, the primary host, has fleshy fruits that attract frugivores, providing food for birds. This may facilitate long dispersal of mistletoes; however, evidences is rarely reported (Watson 2011).

Using methods of spatial analysis, it is possible to describe variation in the spatial distribution and seed dispersal of some types of mistletoes and the scales at which these changes occur (Overton 1994; Aukema 2004). For example, Overton (1996) and Lavorel et al. (1999) developed spatially structured models of mistletoe distributions. Some bird-dispersed 
mistletoes (Amyema miquelii, Dicaeum hirundinaceum) were found to have a leptokurtic (short-distance seed dispersal from the parent plant) seed shadow based on dispersal probability to different distances from the host (Ward and Paton 2007). Spatial patterns of a parasitic root plant (Santalum lanceolatum) have been evaluated using Ripley's $K$ function and 0 -ring statistics to determine dispersion patterns of the parasitic plant, and host-parasite spatial association was analysed by random labelling (Watson et al. 2007). Shaw et al. (2005) used second-order statistics to examine the spatial interactions between healthy trees and Arceuthobium tsugense-infected trees and tested via Monte Carlo simulations to identify whether the infections are sources of spreading. Watson (2009) suggested that variation in host quality (access to water, nutrients and other resources that limit the host) influences the non-random dispersion of parasitic plants in many systems.

The purpose of this study was to answer the question: Is the observed pattern of mistletoe infections compatible with a combined ballistic and bird seed dispersal? Therefore, we examined whether the dispersal of $A$. oxycedri reflects solely ballistic dispersal mode, defined by a specific dispersion pattern, or whether it is related to 'epizoochory', as revealed by the observed spatial pattern of dispersal. For this, we generated dispersal patterns of mistletoe from a spatially explicit null model to simulate different spread scenarios and compare model results with the observed pattern. The work is a step in the construction of null models to test hypotheses of dispersal of parasitic plants.

\section{Materials and methods}

\subsection{Study site and data collection}

Data were collected from two plots in the protected area of the 'Cuenca alta del Manzanares' located in the northwest of the Community of Madrid, Spain (Fig. 1a). Arceuthobium oxycedri was found parasitizing Juniperus oxycedrus in this area (Zavala and Zavala 1993). The first plot (hereafter 'Al') was a 24.98 ha, near the centre of the park, located at 1000 m.a.s.l, with high capacity for local agricultural production and poor natural condition (production farming activities are allowed in this area). Juniperus oxycedrus was strongly affected by the dwarf mistletoe in AI. Here, J. oxycedrus was the third most abundant tree species after Quercus ilex and Cistus Iadanifer. We mapped 126 J. oxycedrus trees in this plot.

The second plot 'All' covered 26.05 ha of a 'farming area', located to the northeast of the park at an average altitude of $1000 \mathrm{~m}$. Juniperus oxycedrus was less affected in plot AII where this species is the most dominant tree. We mapped 234 trees in this plot.

The boundaries of the two plots were mapped, along with the location of each individual host tree (Fig. 1b, c). In each plot, we examined host trees and recorded the presence or/absence of mistletoe infection. Fifty-seven and forty infected trees were found in AI and All, respectively (Table 2). The coordinates for area boundaries and trees were obtained using the differential global positioning system and then converted to the Universal Transverse Mercator (UTM) geographic coordinate system UTM - WGS84.

\subsection{Spatial analysis}

\subsubsection{Univariate spatial point pattern analysis}

We describe the spatial arrangement of individuals and tested hypotheses about spatial pattern, with Ripley's function $K(r)$, nearest-neighbour function $G(r)$ and pair correlation function $g(r)$.

Ripley's $K$ function (Ripley 1977) is a technique for assessing within-patterns point interactions at multiple scales through the analysis of a wide range of within-pattern point distances (Diggle 1983; Cressie 1991). The function has been used in various forest spatial pattern analyses (Stoyan and Penttinen 2000; Dale and Powell 2001). $K(r)$ function is the expected number of points in a circle of radius $r$ centred on an arbitrary point, divided by the intensity of the pattern (Watson et al. 2007). Ripley (1976) proposed an estimator of the $K$-function:

$\hat{K}(r)=\frac{|A|}{\mathrm{n}^{2}}\left[\sum \sum_{i \neq j} \frac{I_{d}\left(d_{i, j}\right)}{W_{i j}}\right]$

where $r$ is the radius of a circle centred on a point in the pattern, $\mathrm{n}$ is the number of points in region $A$ with area $|A|, d_{b j}$ is the Euclidean distance between the $i$ th and $j$ th points, the weight $w_{i j}$ corrects for edge effects and $I_{d}$ is a function that equals 1 if the distance $d_{i, j} \leq r$, otherwise $I_{d}=0$. To remove scale dependence and stabilize variance, a square root transformation can be used (Besag 1977):

$L(r)=\sqrt{\frac{K(r)}{\pi}}-r$

where $L(r)>0$ indicates a clumped pattern up to $r$ and $L(r)<0$ indicates over-dispersion or even distribution up to $r$.

A problem with the $K$-function can be traced to the cumulative nature of the statistics; effects at short scales obscure the effects at broader scales (Getis and Franklin 1987; Wiegand and Moloney 2004; Loosmore and Ford 2006; Wiegnad and Moloney 2014). To overcome this limitation, the pair correlation function $g(r)$, a non-cumulative function that uses only points separated at $r$ distance from each other, provides a better pattern description across scales and identifies the precise scales where significant point-point interactions occur. The relationship between $K$-function and pair correlation function 

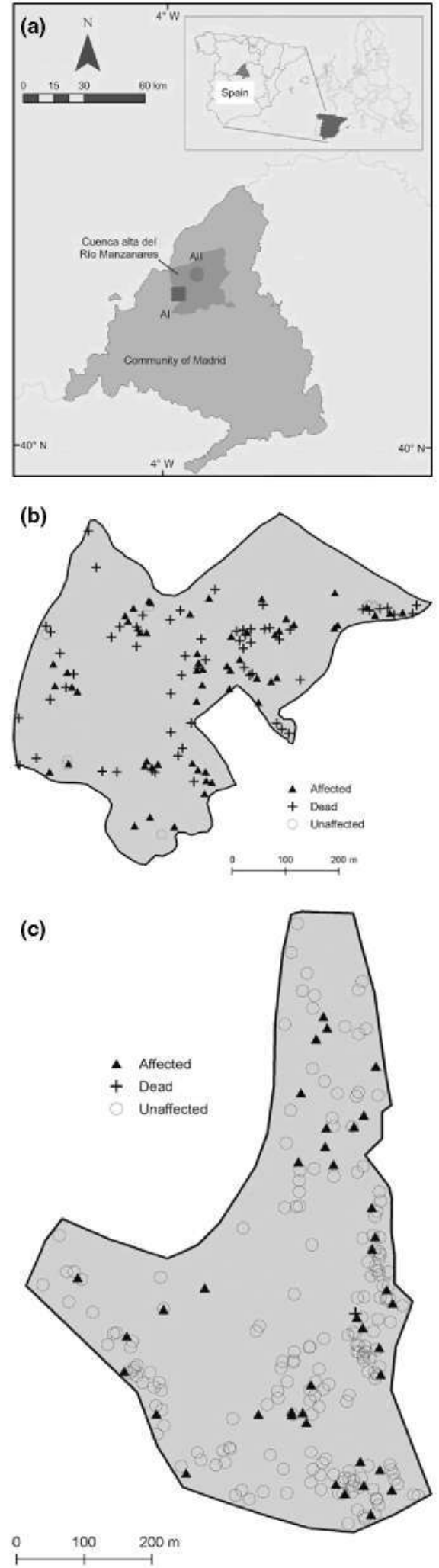

Fig. 1. Spatial distribution of Arceuthobium oxycedri parasitizing Jumiperus oxycedrus in two sampling areas. (a) Site in the Regional Park of Manzanares river upstream, Madrid-España. (b) Sampling area AI and (c) Sampling area AII.

can be expressed as $d K(r) / d r=2 \pi r g(r)$ (Ripley 1977; Stoyan and Stoyan 1996). Both $K(r)$ and $g(r)$ are based on the distribution of distances of all pairs of points, and use the information on all interpoint distances (Ripley 1976, 1977; Diggle 1983; Wiegand et al. 2007). 
Nearest-neighbour analyses provide a useful complement to the second-order statistics described above (Wiegnad and Moloney 2014). The distribution function of nearest-neighbour distances, denoted by $G(r)$ (Diggle 2003), represents the cumulative distribution of the distances $r$ to the nearest neighbour, measured from the typical point of the pattern (Dixon 2002a; Illian et al. 2008). For a pattern with complete spatial randomness (CSR), its expectation is:

$G(r)=1-\exp \left(-\lambda \pi r^{2}\right)$

where $\lambda$ is the intensity of points. $G(r)$ provides information on the local neighbourhood (Wiegand et al. 2013). This property is desirable for specific ecological questions such as the distribution of seedlings around parent trees (Getzin et al. 2008). In a first exploratory analysis, we used the $K$-function to describe the spatial pattern of juniper trees in each site (i.e. we conducted a CSR test. Figure 3 next section), and we also conducted a test of random labelling of infected trees (Watson et al. 2007). We subsequently employed all the functions $(K, g$ and $G$ ) to describe the spatial pattern of infected trees in both study sites and to assess the efficiency of different models in describing the observed patterns.

\subsubsection{Multivariate spatial point pattern analysis}

Bivariate statistics are used to analyse the spatial association of two objects. Here, we consider the multivariate point pattern that arises from the original univariate pattern of trees marked by two host states, infected and uninfected. The spatial dependence between types (e.g. between infected and uninfected hosts) in a multivariate point process is related to second-order properties of multivariate point pattern, which are estimated by 'cross-type' second-moment measures (Cressie 1991). One of the most frequently employed is the 'cross-type' or multitype $K$-function (Hanisch and Stoyan 1979; Diggle 2003). An extension of Ripley's estimator is given by (Dixon 2002b):

$\hat{K}_{i j}(r)=\left(\hat{\lambda}_{i} \hat{\lambda}_{j} A\right)^{-1} \sum_{k} \sum_{f} W\left(i_{k}, j_{i}\right) I\left(d_{i_{k}, j}<r\right)$

where $\lambda_{i}$ and $\lambda_{j}$ are, respectively, the intensity of patterns $i$ and $j, w(i, j)$ is the fraction of the circumference of a circle centred at the $k$ th location of process $i$ with radius $d$ that lies inside the study area. For bivariate analyses, $L_{i j}(r)$ indicates attraction, repulsion or independence of one pattern with respect to a second pattern (in this case, the distribution of infected hosts with respect to uninfected hosts). The dependence scale is determined as the distance at which the peak values of all $L_{i j}(r)$ area are achieved (Lit et al. 2007). Here, we consider the multivariate point pattern marked by infected hosts, and we employ the multitype $K$ to analyse the interaction between infected and uninfected trees.

\subsubsection{Null models and simulation}

To make inferences about mistletoe dispersal, a spatially explicit model (SEM) was defined, based on the following assumptions: (1) all individuals of the host species had the necessary qualities to be infected; (2) the probability of being both infected and uninfected depends on the local densities of plants. Plants live in a two-dimensional space large enough for edge effects to be small. The landscape was assumed homogeneous, that is local host dispersal and interactions between individuals influence spatial structure of mistletoe.

We assume that the distribution of $A$. oxycedri is the consequence of its dispersal mechanism. To approach this dispersal, we use the dispersal kernel defined as the probability density function of dispersal distances from individual plants (Ribbens et al. 1994). We consider two kernel functions: the bi-dimensional 2Dt (fat-tailed, Appendix S1) and the Gaussian (thin-tailed), proposed by Clark et al. (1999). The Gaussian kernel is a reasonable model for a restricted set of conditions, being most sensitive to seeds dispersed over short distances and less suitable to describe sporadic seed dispersed over long distances (Clark et al. 1999). The (reparametrized) formula for this function is:

$k(r)=\left(\pi \sigma^{2}\right)^{-1} \exp \left(-\frac{r^{2}}{\sigma^{2}}\right), \sigma>0$

where $\sigma$ is the standard deviation of the isotropic Gaussian smoothing kernel and $r$ the dispersal distance.

On the other hand, the 2Dt-function allows fitting short and long distance, associated with different dispersal mechanisms. The (reparametrized) formula becomes (Levin et al. 2003):

$P(r)=\left\{\begin{array}{lr}a\left(1+b r^{2}\right)^{-\alpha}, & \text { if } r \leq 10 \\ 0 & \text { if } r>10\end{array}\right.$

where $a$ is a scale parameter, $b$ is a shape parameter and $r$ is the dispersal distance. The parameters $\sigma$ (in Gaussian kernel) and $a$ (in 2Dt kernel) control the bandwidth or smoothing parameter. A detailed description of the two kernel functions proposed by Clark et al. (1999) is presented in Appendix S1.

To simulate spatial patterns generated by dispersal limitation (dispersal described by Gaussian and 2Dt kernels), we started with a configuration of 5, 10 and 20 infected trees in each site. This configuration includes bandwidth values of 10 , 20 and $30 \mathrm{~m}$ for each initial population of infected trees. In our study, the Gaussian and 2Dt models were evaluated at short distance (acting as ballistic dispersion) and large distance (acting as ballistic + bird dispersion). As ballistic seed dispersal of dwarf mistletoes reach a mean distance of $10 \mathrm{~m}$ (Turrill 1920; Maloney and Rizzo 2002), to model ballistic 
Table 1. Parameter values of the simulation models.

\begin{tabular}{|lcc|}
\hline Kernel names & Parameter & Tail fatness \\
\hline Gaussian & $\sigma=5,10$ and $15 \mathrm{~m}$ & Thin-tailed \\
2Dt & $a=10^{1}, 20$ and $30 \mathrm{~m} ; b=2$ & Fat-tailed \\
${ }^{1}$ Common horizontal distances for ballistic seed dispersal of dwarf mistletoe. & \\
\hline
\end{tabular}

dispersal we assigned a probability of dispersal $=0$ for distances larger than $10 \mathrm{~m}$, forcing the null model to consider only a ballistic dispersal mechanism. Values for the parameters of models in the different scenarios are shown in Table 1.

Once we had set the initial distribution of infected trees, we simulated successive dispersal steps. For each simulation, we first built a map of dispersal probability, based on the coordinates of the infected trees and the dispersal kernels. From this map of dispersal probability, we assigned to each healthy tree a probability of infection $\left(p_{i}\right)$. Finally, we assigned the status infected/healthy to each individual as a Bernoulli trial with $p=p_{i}$.

We repeated the same process (construction of new dispersal probability maps and assignment of new infected status), while the number of infected trees in the simulated pattern was smaller than the infected trees in the observed pattern.

For each kernel (Gaussian and 2Dt), we evaluated nine scenarios, resulting from the combination of three different initial numbers of infected trees (5,10 and 20 individuals) and three different bandwidths ( $a=10,20$ and $30 \mathrm{~m}$ ) for the $2 \mathrm{Dt}$ and the equivalent for the Gaussian kernel (i.e. $\sigma=5,10$ and $15 \mathrm{~m}$ ). The $r$ distance interval was maintained from 0 to $125 \mathrm{~m}$.

\subsubsection{Evaluation of the simulated models}

To assess differences between the spatial structure of the observed infected trees and the predictions from each model, we computed Ripley's $K$ function, pair correlation function $g(r)$ and nearest-neighbour distance distribution function $G(r)$ on the pattern of infected trees in each study site. We also computed simulation envelopes based on the simulated models. For each scenario (i.e. combination of initial number of infected trees and kernel bandwidth) and study site, we simulated 199 patterns and also computed the functions $K, g$ and $G$. From the set of 'simulated' functions we build for each function, the simulation envelopes which included the highest and lowest simulated values. Significant departures from the simulated model were identified at $r$ distance, when the empirical function $(K, G$ or $g$ ) was located outside of the simulation envelopes. Thus, if ballistic dispersal is prevalent, we would not expect significant differences at short scales $r$ with Gaussian kernel and all values of scale parameter (sigma). In another way, if the mixture of ballistic and other forms of longdistance dispersal prevail, differences at medium and large scales $r$ with 2Dt kernel would not be expected.

We also computed the goodness-of-fit $(\mathrm{GoF})$ test proposed by Loosmore and Ford (2006). Both the observed pattern and each of the patterns simulated by the null model were reduced to a single statistic $\left(u_{i}\right)$ as a distance function, representing the total squared deviation between the observed pattern and theoretical results over the distance r. For our objectives of evaluating the fit of models to the observed data, the relevant tests are those showing non-significant values for the u statistic (i.e. absence of significant deviations from the expected values under the null model).

All calculations were conducted using the program R ( $R$ Core Team 2013). Spatial analysis and model fitting were computed using the R packages 'Spatstat' (Baddeley and Turner 2005) and 'ecespa' (De la Cruz 2008).

\section{Results}

\subsection{Spatial distribution of Arceuthobium oxycedri}

We classified Juniperus oxycedrus in three categories: dead, affected and unaffected. Affected host plants occurred unequally in the two sampling areas, $45.3 \%$ in AI and $17.1 \%$ in AII. Unaffected hosts were 6.3 and $82.5 \%$ in AI and AII, respectively (Table 2). We recorded a high mortality in Al $(48.8 \%)$ and $<1 \%$ in All. These differences were significant $\left(\chi^{2}=218.6\right.$, df $=$ 2 , p-value $<0.0001$ ), revealing a different distribution of marks between the two sampling areas (Fig. 2). The underlying pattern intensity of infected hosts differed between the two areas: in AI, the average distance to the nearest neighbour was $22.2 \mathrm{~m}$, while in All, the distance was $37.1 \mathrm{~m}$. In the case of uninfected hosts, these distances were $82.5 \mathrm{~m}$ in $\mathrm{Al}$ and $14.6 \mathrm{~m}$ in All.

Tabie 2. Observed frequency of host and parasite in the studied plots (luniperus oxycedrus/Arceuthobium oxycedri).

\begin{tabular}{|lcccc|}
\hline Plot & Affected $(A)$ & Unaftected $(N)$ & Dead $(M)$ & Total $(\mathrm{n})$ \\
\hline AI & $57(45.3 \%)$ & $8(6.3 \%)$ & $61(48.4 \%)$ & 126 \\
AII & $40(17.1 \%)$ & $193(82.5 \%)$ & $1(0.4 \%)$ & 234 \\
\hline
\end{tabular}




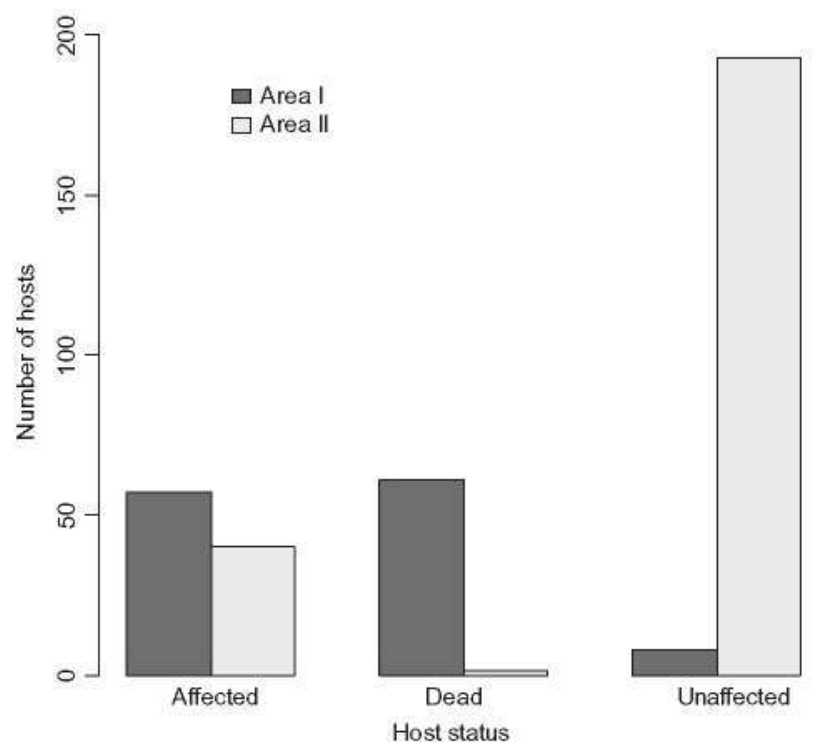

Fig. 2. Numbers of Juniperus oxycedrus with Arceuthobium oxycedri infections, mortality due to mistletoe infection, or uninfected in to the two sampling areas.

\section{1 .1 Univariate and multivariate spatial point pattern analysis}

The host distributions showed strong aggregation in AI at scales from $20 \mathrm{~m}$ to $130 \mathrm{~m}$ and aggregation at all distances in AII, although slight clustering with random tendency at short distance (Fig. 3). The spatial pattern of infected trees was apparently random in both sampling sites (Fig. 4).

The patterns of infected and uninfected trees were independent (Fig. 5) although a moderate attraction between infected and uninfected trees from $0 \mathrm{~m}$ to $80 \mathrm{~m}$ (i.e. in this scale, infected trees were surrounded by uninfected trees), and repulsive tendency (i.e. more often infected trees were surrounded by infected trees) at large distances was evident in AI (Fig 5 a). In AII, dependence between infected and uninfected tree patterns was not evident, for all distances (Fig. 5b).

\subsection{Simulating dispersal scenarios}

As the scenarios for $\sigma=5 \mathrm{~m}$ did not converge (i.e. were unable to simulate the spread of the infection from the initial populations), only six scenarios could be analysed for the Gaussian kernel.

Among the six Gaussian models that converged in plot AI, most showed significant differences with the observed patterns (Fig. 6; Table 3a). However, the models that included large bandwidths $(\sigma=15 \mathrm{~m}$, i.e. $h=30 \mathrm{~m})$ and medium and large original populations of infected trees (i.e. $P_{0}=10$ and 20 individuals) were able to simulate the original patterns, that is there were no significant departures of the observed $K, g$ and $G$ functions from the simulation envelopes (Table $3 a$ ).

In the case of the 2Dt models, the results depended mostly on the summary function. For example, none of the models appropriately described the observed pair correlation function $g(r)$ (Table 4a). However, the $K$-function was within the envelopes for all models (i.e. all bandwidths) simulated with an original population of $P_{0}=10$, and the nearest-neighbour
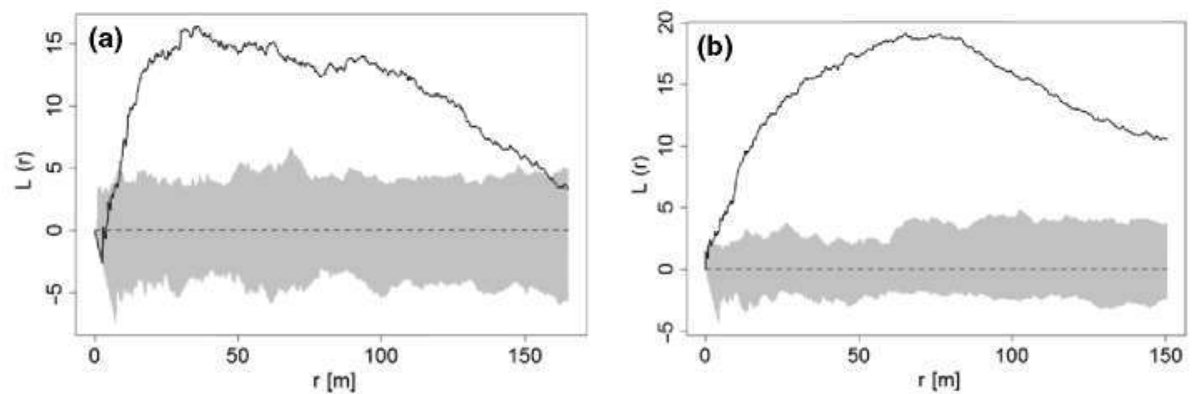

Fig. 3. Aggregation differences between host trees in both plots. The solid line represents the empirical values of $L(r)$ and the dashed line represents homogeneous Poisson process. The grey region represents the $99 \%$ confidence envelops constructed with 99 Monte Carlo simulations $K$-functions of the Arceuthobium oxycedi hosts point patterns assuming homogeneous Poisson process (red dashed line). (a) Plot AI, (b) Plot AII. 

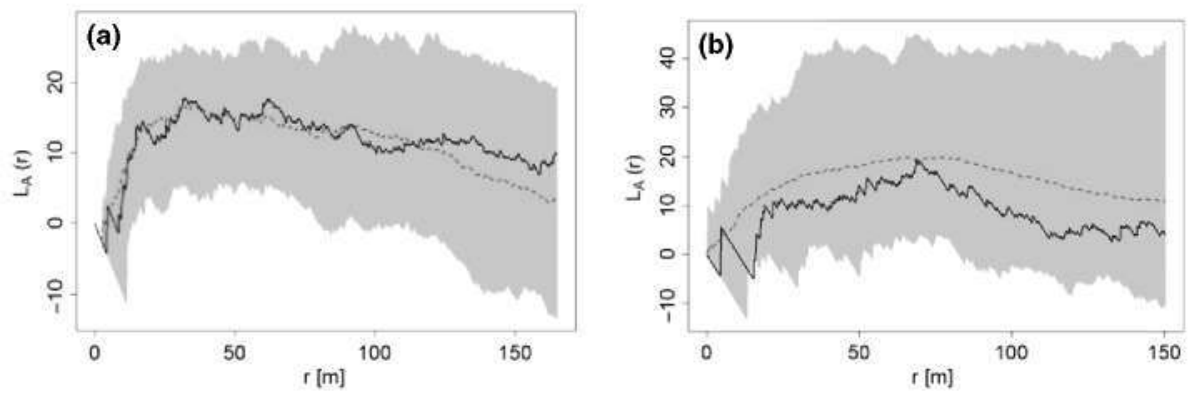

Fig. 4. Test of random labelling of the spatial pattern of infected host trees in plot AI (a) and plot AII (b). The solid lines represent the empirical values of $L(r)$, dotted red line represents the average values of $L(r)$ and the grey region represents $99 \%$ confidence envelopes constructed from 199 relabellings of the infected marks on the whole population of trees in each area.
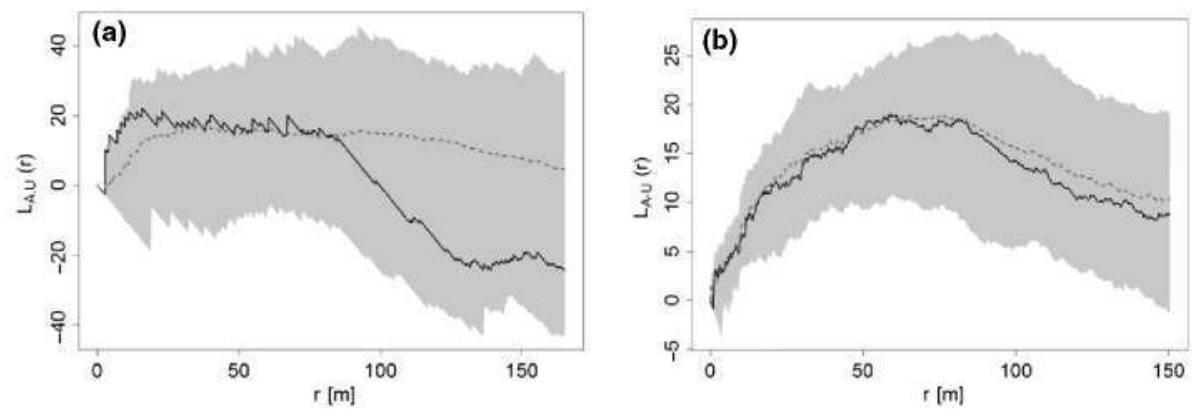

Fig. 5. Test of association between infected (a) and uninfected (U) Juniperus oxycedrus trees with random labelling. The solid lines represent the empirical values of $L_{\mathrm{A}, \mathrm{U}}(r)$, dotted red line represents the average of random labelling simulations and the grey region represents $99 \%$ confidence envelopes constructed from 199 relabellings of the infected marks on the whole population of trees in each area (a) Plot AI, (b) Plot All.
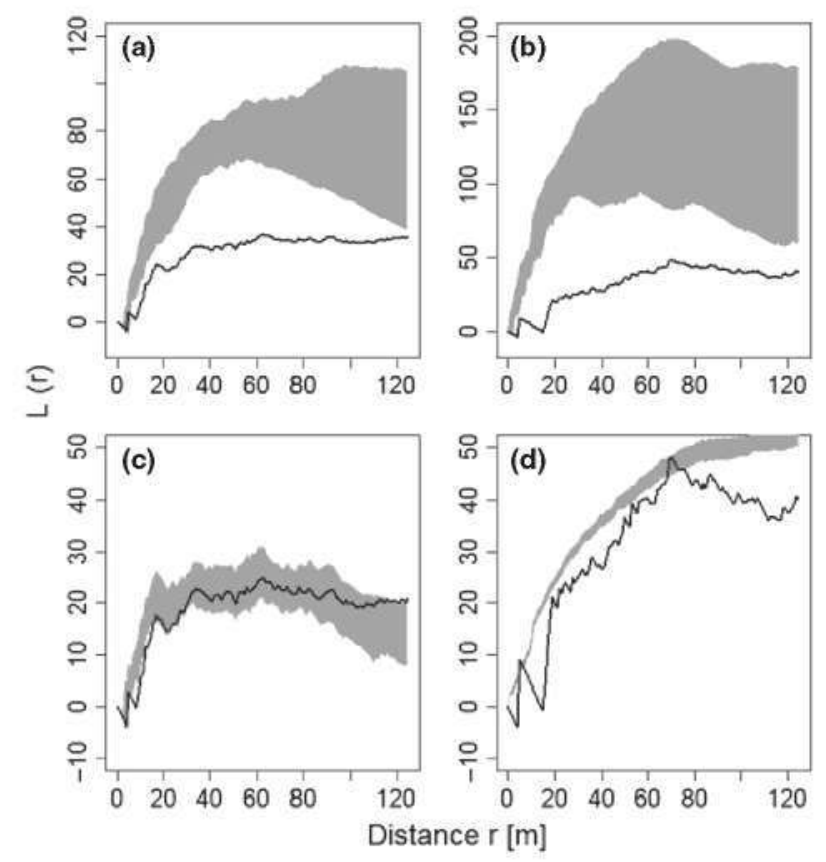

Fig. 6. Envelopes for the $K$-function from the simulation of different dispersal patterns of Arceuthobium oxycedri. (a, b) Null models with Gaussian kernel for AI and AII plots, respectively. (c, d) Null models with 2Dt kernel, with $P_{0}=10, \sigma=5 \mathrm{~m}$ (Gaussian) and $a=10 \mathrm{~m}$ (2Dt), for AI and AII plots, respectively. The solid lines are empirical values of $L(r)$, and the grey region represents $99 \%$ confidence envelopes with from 199 simulations of the null model. 
distances distribution $G(r)$ was correctly described for all values of $P_{0}$ (with some restrictions on the value of $h$ in some cases) (Table 4a).

In the plot AII, only the Gaussian model (with $P_{0}=20$ ) was able to describe the pattern of infected trees, and only for the $K$-function (Table $3 b$ ). On the contrary, none of the nine combinations of parameters for the 2Dt models was able to describe any of the summary functions employed (Table 4b).

Table 3. GoF test for the null Gaussian models of the dispersal pattern of Arceuthobium oxycedri. Where K: Ripley's $K$ function, G: distances to the nearest-neighbour distribution, $g$ : pair correlation function. (a) Area I (b) Area [l. $P_{0}$ : initial infected population, $\sigma$ : bandwidth.

\begin{tabular}{|c|c|c|c|c|}
\hline \multirow[b]{2}{*}{$P_{0}$} & \multirow[b]{2}{*}{$\sigma$} & \multicolumn{3}{|c|}{ GoF test (p-value) } \\
\hline & & $K$ & G & $g$ \\
\hline \multicolumn{5}{|l|}{ (a) } \\
\hline \multirow[t]{3}{*}{5} & 10 & - & - & - \\
\hline & 20 & - & - & - \\
\hline & 30 & - & - & - \\
\hline \multirow[t]{3}{*}{10} & 10 & $\neq * *$ & $* \neq *$ & *** \\
\hline & 20 & $\neq$ & **** & *** \\
\hline & 30 & ns & ns & ns \\
\hline \multirow[t]{3}{*}{20} & 10 & * & **** & *** \\
\hline & 20 & $\neq$ & **** & * \\
\hline & 30 & ns & ns & *** \\
\hline \multicolumn{5}{|c|}{ (b) } \\
\hline \multirow[t]{3}{*}{5} & 10 & $\neq \neq$ & $* \neq *$ & *** \\
\hline & 20 & * & $* * *$ & * \\
\hline & 30 & * & **** & * \\
\hline \multirow[t]{3}{*}{10} & 10 & $=$ & **** & ** \\
\hline & 20 & * & *** & ** \\
\hline & 30 & * & **** & $*$ \\
\hline \multirow[t]{3}{*}{20} & 10 & $\dagger$ & $* \neq *$ & ** \\
\hline & 20 & ns & **** & * \\
\hline & 30 & ns & $* * *$ & * \\
\hline
\end{tabular}

Table 4. GoF test for the null 2Dt models of the dispersal pattern of Arceuthobium oxycedri. Where (a) Area I and (b) Area Il. $P_{0}$ : initial infected population, $\sigma$; bandwidth.

\begin{tabular}{|c|c|c|c|c|}
\hline \multicolumn{5}{|c|}{ 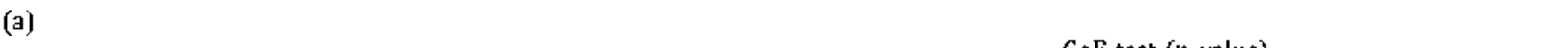 } \\
\hline$P_{0}$ & $\sigma$ & $K$ & $G$ & $g$ \\
\hline \multirow[t]{3}{*}{5} & 10 & $*$ & $*$ & *** \\
\hline & 20 & $* * *$ & $*$ & $* * *$ \\
\hline & 30 & $*$ & $\uparrow$ & $* * *$ \\
\hline \multirow[t]{3}{*}{10} & 10 & $\dagger$ & ns & *** \\
\hline & 20 & ns & ns & *** \\
\hline & 30 & 9 & ns & $* * *$ \\
\hline \multirow[t]{3}{*}{20} & 10 & $*$ & ns & $* * *$ \\
\hline & 20 & $*$ & ns & **** \\
\hline & 30 & $*$ & $*$ & $* * *$ \\
\hline \multicolumn{5}{|l|}{ (b) } \\
\hline & & \multicolumn{3}{|c|}{ GoF test (p-value) } \\
\hline$P_{0}$ & $h$ & $K$ & $G$ & $g$ \\
\hline \multirow[t]{3}{*}{5} & 10 & $\neq \neq *$ & $\neq * *$ & *** \\
\hline & 20 & **** & $* * *$ & *** \\
\hline & 30 & $* * *$ & $* * *$ & *** \\
\hline \multirow[t]{3}{*}{10} & 10 & $\neq \neq *$ & $\neq * *$ & *** \\
\hline & 20 & $\neq \neq *$ & $\neq * *$ & *** \\
\hline & 30 & $* * *$ & $* * *$ & *** \\
\hline \multirow[t]{3}{*}{20} & 10 & $\neq \neq=$ & $\neq * *$ & *** \\
\hline & 20 & $\neq \neq *$ & $\neq * *$ & *** \\
\hline & 30 & $* * *$ & $* * *$ & $* * *$ \\
\hline
\end{tabular}




\section{Discussion}

\subsection{Mistletoe distribution}

Junipers (uniperus oxycedrus) provide a suitable habitat for protection, nourishment and reproduction to its obligate parasitic $A$. oxycedri (Sarangzai et al. 2010). In our study sites, the infection process varied across sites, although it was more localized in Al. Prevalence infection rates were 45 and $17 \%$, in Al and All, respectively. In Al, Juniperus was not the dominant genus, but was dominant in All where there was no significant woody understory (Zavala and Zavala 1993). Host community composition can fundamentally influence the establishment and prevalence of infection (Holt et al. 2003), and different factors may influence mistletoe aggregation in opposing directions sometimes acting to reduce the overall levels of aggregation (Rist et al. 2011). In plots examined here, the average nearest-neighbour distance of trees infected was greater in All (37.1 m) than in AI (22.2 m). Density of the hosts could be a factor that explains the prevalence of infection, as a distinctive feature of dwarf mistletoes is seed dispersal over short distances (Robinson and Geils 2006). This effect, added to host availability, may lead to an aggregated distribution of mistletoes. The spatial pattern of hosts plays an important role in intensification events defining severity within the host tree (Shaw et al. 2005). This effect, combined with the different levels of disturbance in the two plots, explains in part the difference in spatial pattern of infected trees. Interactions of trees with different marks (affected hosts vs. unaffected hosts) occurred at all distances including short-range seed dispersal associated with the ballistic mode $(<10 \mathrm{~m})$. This analysis revealed a strong dependence between infected and uninfected host point patterns, indicating that uninfected trees tend to be co-located within $100 \mathrm{~m}$ of each other in plot AI (150 $\mathrm{m}$ in plot AII) of an arbitrary affected host, indicating local infection and aggregated distribution of mistletoe. Spatial heterogeneity in host tree susceptibility may also underlie mistletoe distribution (Ward and Paton 2007); in the present work, the most infected and less vigorous hosts were observed in plot AI.

\subsection{Simulation models}

\subsubsection{Mistletoe simulated patterns vs. observed pattern}

The density of infected trees was estimated using a nonparametric model based on kernel function, which is relevant to seed dispersal or epidemiological studies (Brown and Bolker 2004; Carlo and Morales 2008). The kernel estimator is arguably the most popular nonparametric estimator of density currently used (Marchette 2009). Although seed ballistic horizontal displacement of Arceuthobium spp. can be up to $16 \mathrm{~m}$, a horizontal average distance of $10 \mathrm{~m}$ from the mother plant is more common (Hawksworth et al. 2002). In most scenarios, the observed spatial structure of Arceuthobium infections was different from each of the simulated models based exclusively on ballistic dispersal (Gaussian kernel, bandwidth $=5 \mathrm{~m}$ ). This discrepancy suggests that other agents may influence dispersion of $A$. oxycedri, for example a combinations of ballistics and birds. The Gaussian model with $30 \mathrm{~m}$ bandwidth I explained the spatial pattern in plot AI, whereas $20 \mathrm{~m}$ and smaller bandwidths could not. In plot AII, only the Gaussian null model generated consistent simulations, for bandwidths from 10 to $30 \mathrm{~m}$. This large bandwidth suggests that additional factors besides the ballistic dispersion influencing mistletoe dispersal. On the other hand, although host tree aggregation at smaller spatial scales was more evident in AII than in Al, the clustering of infected tress was not significantly different in both areas. This result suggests that patterns of infection in plot AI may have resulted from positive feedback in which high mistletoe prevalence leads to increased seed deposition in uninfected trees (Aukema 2004). Shaw et al. (2005) examined the spatial association between healthy and infected Arceuthobium tsugense trees, and identified a negative association whereby infected trees were aggregated and repelled from uninfected trees. In our case, slight repulsion between marks (infected/uninfected) was found in plot Al, occurring from $80 \mathrm{~m}$; however in plot AII, this association was neutral.

According to the distribution function of nearest-neighbour distances, in $95 \%$ of cases, the nearest infected tree (to a given infected tree) was $<50 \mathrm{~m}$ away in Al and $80 \mathrm{~m}$ away in All (Fig. 7). Pair correlation function showed high values at fines scales (i.e. distance next to zero), which was indicative of clustering (Stoyan and Stoyan 1996), suggesting that ballistic dispersal was the predominant dispersal vector of $A$. oxycedri and probably acts to intensify the infection within the host tree (Fig. 8).

In plot AII, significant differences between observed pattern of infected trees and simulated patterns were more consistent for all scenarios. It is important to consider that estimated values of the $K$-function, in contrast to the pair correlation function, complicate the interpretation when clustering is stronger at small scales (Wiegnad and Moloney 2014) as high density at small scales influences the values of the $K$-function at larger scales. This effect is called 'memory' of the $K$-function (Wiegand and Moloney 2004). However, spatial structural variability in both plots suggested that infection was present in plot AI before All. The higher proportions of trees affected and dead in AI in relation to plot AII also support this conclusion. Clustering over $10 \mathrm{~m}$ indicated patchiness at a broad spatial scale, which can be attributed to the presence of additional factors for dispersal including birds, mammals and insects (Gajšek et al. 2013). The difference in aggregation between plots may have several causes, such as microhabitat suitability, habitat heterogeneity (including local disturbances) and biotic interactions (Qeijeiro-Bolaños et al. 2014).

Watson (2009) suggested that variation in host quality influences the non-random dispersion of parasitic plants in many systems. The environmental differences between AI and AII could be affecting the vigour of $l$. oxycedrus individuals differently and this could be reflected in a differential dispersal of $A$. oxycedri in each area. In plot AI, affected trees had 

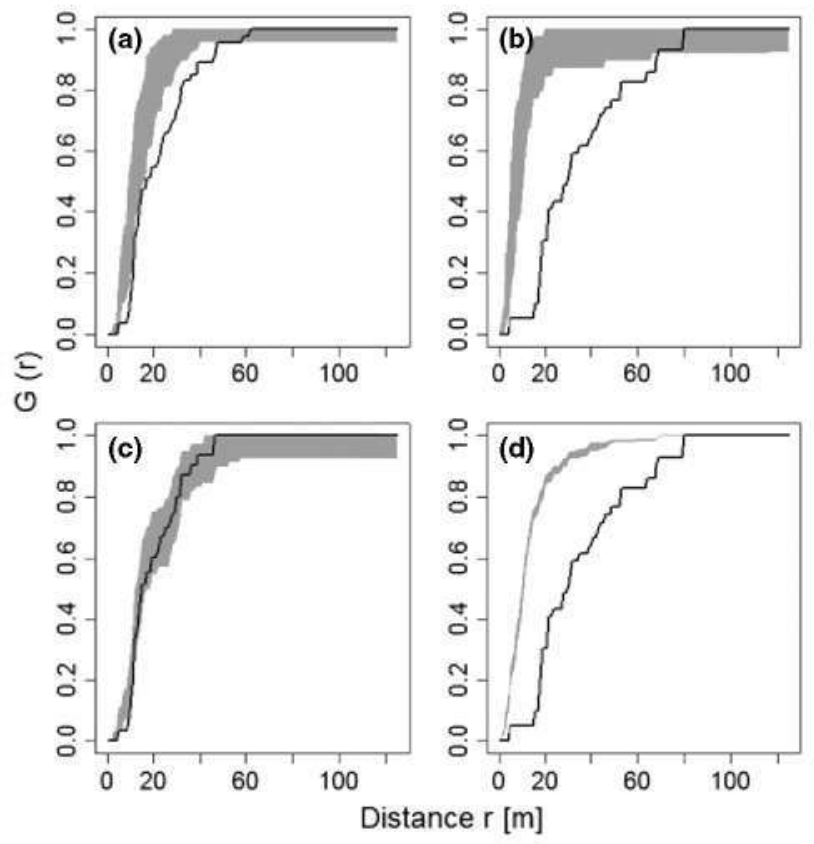

Fig. 7. Envelopes for the $G$-function from the simulation of different dispersal patterns of Arceuthobium oxycedri. (a, b) Null models with Gaussian kernel for AI and AII plots, respectively. (c, d) null models with 2Dt kernel, with $P_{0}=10, \sigma=5 \mathrm{~m}$ (Gaussian) and $a=10 \mathrm{~m}$ (2Dt), for AI and AII plots, respectively. The solid lines are empirical values of $G(r)$, and the grey region represents $99 \%$ confidence envelopes with from 199 simulations of the null model.
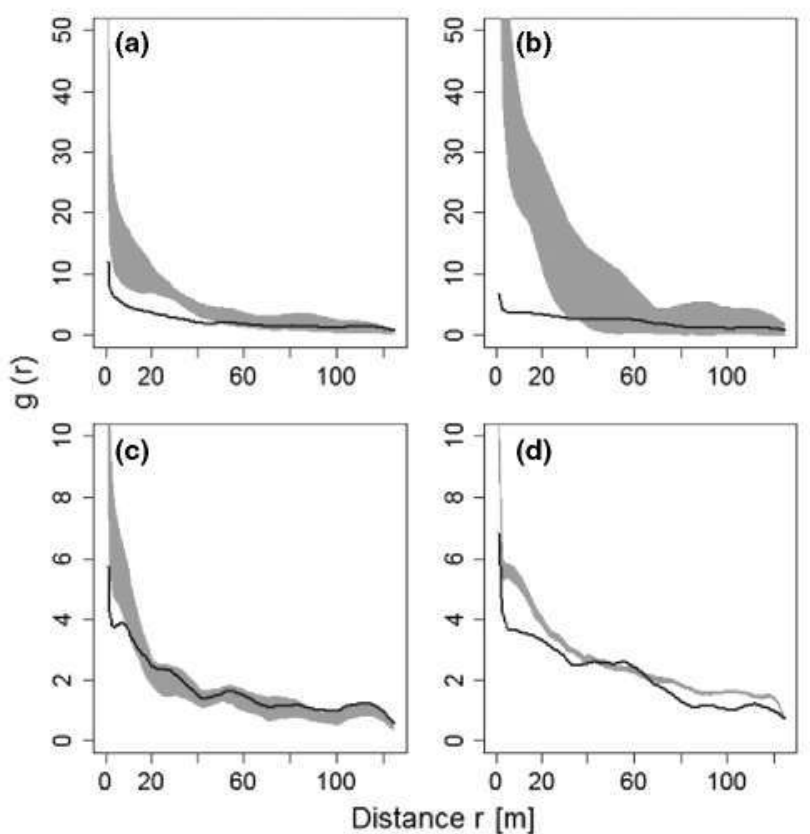

Fig. 8. Envelopes for the pair correlation function $(g)$ from the simulation of different dispersal patterns of Arceuthobium oxycedri. (a, b) null models with Gaussian kernel for AI and AII plots, respectively. (c, d) null models with 2Dt kernel, with $P_{0}=10, \sigma=5 \mathrm{~m}$ (Gaussian) and $a=10 \mathrm{~m}(2 \mathrm{Dt})$, for $\mathrm{AI}$ and AII plots, respectively. The solid lines are empirical values of $g(r)$, and the grey region represents $99 \%$ confidence envelopes with from 199 simulations of the null model.

below-average vigour and were heavily infected, while in plot AII, trees with average vigour were affected in a different way by the dwarf mistletoe (Zavala and Zavala 1993).

Despite the ballistic dispersion form, the pattern of infected trees shows a larger range of distances to the nearest neighbour than expected, that is infected trees are more scattered than would be expected by only ballistic dispersion as modelled by the Gaussian null model, thereby suggesting the implication of another vector, such as birds dispersals (Sarangzai et al. 2010). Dwarf mistletoe recruitment may require restricted safe sites; therefore, specialized frugivores may be 
advantageous with respect to ballistic dispersal specially for colonizing new hosts. Recently, Mellado and Zamora (2014) have shown that as much as eleven bird species can disperse mistletoe seeds of the European mistletoe Viscum album subsp. austriacum. As birds behave similarly on parasitized and non-parasitized hosts, and birds exhibit extensive ranges, an ample assemblage of generalist birds ensures both reinfection within patches and the colonization of new patches. This suggests that while ballistic dispersal might be advantageous for local colonization of infected trees, bird dispersal - even with unspecialized dispersers - might enhance both local and external colonization. Nicholls et al. (1984) observed that migratory birds are more important in long-distance spread of dwarf mistletoes.

Our sites reveal a variable fraction of host mortality, but Juniper mortality may result from a number of chained events, including predisposing, inciting and triggering factors (Manion 1981). Unravelling the role of dwarf mistletoes as a causal process or as a consequence of other phenomena (e.g. drought) is beyond the aim of this study. Yet spatially explicit dispersal and distribution models can contribute to decision-making processes for forest managers. Management of dwarf mistletoes relies on scientific understanding of the ecology and epidemiology of these important pathogens in the context of on-the-ground forest conditions (Hawksworth and Wiens 1996). Further observational and experimental work is needed to clarify the mechanisms underlying the infection pattern seen. For example, current infection patterns may reflect a legacy from previous demographic or human-induced processes, both in tree spatial distributions and within individual trees. Also host vulnerability may be conditioned by predisposing factors such as soil heterogeneity or microclimate, as well as genetic variability in the host, that combine to result in the observed patterns of infection. Despite these variations, we suggest that spatial null models, in combination with empirical studies, have great potential to increase understanding of the mechanisms underlying dispersal of $A$. oxycedri.

\section{Acknowledgements}

This work was partially supported by A/030244/10 project, financed by Agencia Española de Cooperación Internacional y para el Desarrollo (AECID), project lslas-Espacio CGL2009-13190-C03-02 financed by the Spanish Ministerio de Ciencia, and REMEDINAL 3-CM (CAM S2013/MAE-2719, 2014-2018). Special thanks to Juliann Aukema (Aukema Conservation Science), Pedro Quintana-Ascencio (University of Central Florida) and David Duncan for constructive comments on earlier versions of the manuscript.

\section{References}

Akkol, E. K.; Orhan, I.; Kartal, M.; Yesilada, E., 2010: Bioactivity guided evaluation of anti-inflammatory and antinociceptive activities of Arceuthobium oxycedri (D.C.) M. Bieb. 1. Ethnopharmacol. 128, 79-84.

Aukema, J. A., 2003: Vectors, viscin, and Viscaceae: mistletoes as parasites, mutualists, and resources. Front. Ecol. Environ. 1, $212-219$.

Aukema, J. E., 2004: Distribution and dispersal of desert mistletoe is scale-dependent, hierarchically nested. Ecography 27, 137-144.

Aukema, J. E.; Martínez del Río, C., 2002: Mistletoes as parasites and seed-dispersing birds as disease vectors: current understanding, challenges, and opportunities. In: Seed dispersal and frugivory: Ecology, evolution and conservation. Ed. by Levey, D. J.; Silva, W. R.; Galetti, M. Oxfordshire, UK: CAB International Press, pp. 99-110.

Baddeley, A.; Tumer, R., 2005: Spatstat: an R package for analyzing spatial point patterns. ]. Stat. Softw. 12, 1-42.

Besag, J., 1977: Contribution to the discussion of Dr. Ripley's paper. J. R. Stat. Soc. B 39, 193-195.

Brown, D.; Bolker, B., 2004: The eftects of disease dispersal and host clustering on the epidemic threshold in plants. Bull. Math. Biol. 66, 341-371.

Carlo, T. A.; Morales, ]. M., 2008: Inequalities in fruit-removal and seed dispersal: consequences of bird behaviour, neighbourhood density and landscape aggregation. J. Ecol. 96, 609-618.

Catalán, P. 1997: Arceuthobium. In: Flora Iberica. Plantas vasculares de la Península Ibérica e Islas Baleares (Vol. VIII, HaloragaceaeEuphorbiaceae). Ed. by Castroviejo, S.; Aedo, C.; Benedí, C.; Lánz, M.; Muñoz Garmendia, F.; Nieto Feliner, G.; Paiva, J. Madrid: Real Jardín Botánico, CSIC, pp. 164-166.

Ciesla, W. M.; Geils, B. W.; Adams, R. P., 2004: Host and geographic distribution of Arceuthobium oxycedri. RMRS-RN-11WWW. Fort Collins, CO: U.S. Department of Agriculture, Forest Service, Rocky Mountain Research Station. Available in http: $/ /$ www.fs.fed.us $/ \mathrm{rm} / \mathrm{pubs} /$ rmrs_rn11/.

Clark, J. S.; Silman, M.; Kern, R.; Macklin, E.; HilleRisLambers, 1., 1999: Seed dispersal near and far; patterns across temperate and tropical forests. Ecology 80, 1475-1494.

Cressie, N. A., 1991: Statistics for spatial data. New York: Wiley.

Dale, M. R. T.; Powell, R. D., 2001: A new method for characterizing point patterns in plant ecology. J. Veg. Sci. 12, 597-608.

De la Cruz, M., 2008: Métodos para analizar datos puntuales. In: Introducción al Análisis Espacial de Datos en Ecología y Ciencias Ambientales: Métodos y Aplicaciones. Ed. by Maestre, F. T.; Escudero, A.; Bonet, A. Madrid: Asociación Española de Ecología Terrestre, Universidad Rey Juan Carlos y Caja de Ahorros del Mediterráneo, pp. 76-127. ISBN: 978-84-9849-308-5.

Diggle, P. I. 1983: Statistical analysis of spatial point patterns. Mathematics in Biology. Vol. 2. London, New York: Academic-Press.

Diggle, P. J., 2003: Statistical analysis of spatial point patterns. 2nd edn. London, UK: Arnold.

Dixon, P. M., 2002a: Nearest neighbor methods. Encycl. Environ. 3, 1370-1383.

Dixon, P. M., 2002b: Ripley's $K$ function. Encycl. Environ. 3, 1796-1803.

Gajšek, D; Jami, K.; Brus, R. 2013; Infection patterns and hosts of Arceuthobium oxycedri (DC.) M. Bieb. In Slovenia.. Forest Pathol, 43. $185-192$.

Getis, A.; Franklin, J., 1987; Second-order neighborhood analysis of mapped point patterns. Ecology 68, 473-477.

Getzin, S.; Wiegand, T.; Wiegand, K.; Fangliang, H., 2008: Heterogeneity influences spatial patterns and demographics in forest stands. J. Ecol. 96, 807-920.

Hanisch, K. H.; Stoyan, D., 1979; Formulas for the second-order analysis of marked point processes. Math. Operationsforschung Stat. Ser. Stat. 10,555-560.

Hawksworth, F. G.; Geils, B. W., 1996: "Biotic associates". In: Dwarf mistletoes: Biology, pathology, and systematics. Ed. by Hawksworth, F. G.; Wiens, D. Agricultural Handbook 709. Washington, DC: U.S. Department of Agriculture, Forest Service, pp. 410. 
Hawksworth, F. G.; Wiens, D., 1996: Dwarf mistletoes: Biology, pathology and systematics. Agriculture Handbook 709. Washington, DC: U.S.D.A. Forest Service.

Hawksworth, F. G.; Wiens, D; Geils, B. W., 2002: Arceuthobium in North America. In: Mistletoes of North American conifers. Chapter 4. General Technical Report RMRS-GTR-98. Ed. by Geils, B. W.; Tovar, J. C.; Moody, B. (Tech. Coord.) Ogden, UT United States: Department of Agriculture, Forest Service, Rocky Mountain Research Station.

Holt, R. D.; Dobson, A. P.; Begon, M.; Bowers, R. G.; SChauber, E. M., 2003; Parasite establishment in host communities. Ecol. Lett. 6, 837-842.

lllian, 1.; Penttinen, A.; Stoyan, H.; Stoyan, D., 2008: Statistical analysis and modeling of spatial point patterns. England, UK: lohn Wiley \& Sons Ltd.

Larson, D. L, 1996: Seed dispersal by specialist versus generalist foragers: the plant's perspective. Oikos 76, 113-120.

Lavorel, S. Stafford, M.; Reid, N., 1999: Spread of mistletoes (Amyema reissii) in fragmented Australian woodlands: a simulation study. Landscape Ecol. 14, 147-160.

Levin, S. A.; Muller-Landau, H. C.; Nathan, R.; Chave, J., 2003: The ecology and evolution of seed dispersal: a theoretical perspective. Annu. Rev. Ecol. Evol. Syst, 34, 575-604.

Liu, D.; Kelly, M.; Gong, P.; Guo, Q., 2007: Characterizing spatial-temporal tree mortality patterns associated with a new forest disease. For. Ecol. Manage. 253, 220-231.

Loosmore, N. B.; Ford, E. D., 2006: Statistical inference using the G or K point pattern spatial statistics. Ecology 87, 1925-1931

Maloney, P.; Rizzo, D., 2002: Dwarf mistletoe-host interactions in mixed-conifer forests in the Sierra Nevada. Phytopathology 92, 597-602.

Manion, P. D., 1981: Tree disease concepts. Englewood Clifts, NJ: Prentice-Hall Inc., pp. 409.

Marchette, D., 2009: Filtered kernel density estimation. John Wiley \& Sons, Inc. W]REs Comp Stat 1, 106-10.

Mellado, A.; Zamora, R., 2014: Generalist birds govern the seed dispersal of a parasitic plant witl strong recruitment constraints. Decologia 176, 139-147.

Nathan, R. 2007: Total dispersal kernels and the evaluation of diversity and similarity in complex dispersal systems. ln: Seed dispersal: Theory and its application in a changing world. Ed. by Dennis, A. J.; Schupp. E. W.: Green, R. J.: Westcott, D. W. Wallingford, UK: CAB International, pp. 252-276.

Nicholls, T. H.; Hawksworth, F. G.; Merrill, L. M. 1984: Animal vectors of dwarf mistletoe, with special reference to Arceuthobium americanum on lodgepole pine. In: Hawksworth F.G. and Scharpf R.F. (eds) Biology of Dwarf Mistletoes: Proceedings of the Symposium. USDA For. Serv. Gen. Tecl. Rep. RM-111, pp. 102-110.

Ostry, M. E.; Nicholls, T. H.; French, D. W. 1983: Animal Vectors of Eastern Dwarf Mistletoe of Black Spruce, U.S. Dept. of Agriculture. Forest Service. North Central Forest Experiment Station. Research paper NC-232.

Overton, J. M., 1994: Dispersal and infection in mistletoe metapopulations. J. Ecol. 82, 711-723.

Overton, ]. M., 1996: Spatial autocorrelation and dispersal in mistletoes: field and simulation results. Vegetation 125, 83-98.

Qeijeiro-Bolan̄os, M.; Cano-Santana, Zn; García-Guzmán, G., 2014: Incidence, severity, and aggregation patterns of two sympatric dwarf mistletoe species (Arceuthobium spp.) in Central Mexico. Eur. J. Forest Res. 133, 297-306.

R Core Team 2013. R: A language and environment for statistical computing. Vienna, Austria: R Foundation for Statístical Computing. URL https://www.R-projectorg/

Ribbens, E.; Silander, J. A.; Pacala, S. W., 1994: Seedlings recruitment in forest: calibrating models to predict patterns of tree seedling dispersion. Ecology 75, 1794-1806.

Ríos Insua, V., 1987: Contribución al estudio de la biología de Arceuthobium oxicedri (DC.) M. Bieb. (1819), Bol. Sanid. Veg. Plagas 13, 53-62.

Ripley, B. D., 1976: The second-order analysis of stationary point processes. J. Appl. Probab. 13, 255-266.

Ripley, B. D., 1977: Modelling spatial patterns. J. R. Stat. Soc. Ser. B Methodol. 39, 172-212.

Rist, L.; Shaanker, R. U.; Ghazoul, 1., 2011: The spatial distribution of mistletoe in a Souther lndian tropical forest at multiple scales. Biotropica 43, 50-57.

Robinson, D.; Geils, B., 2006: Modelling dwarf mistletoe at three scales: life history, ballistics and contagion. Ecol. Model. 199, 23-38.

Sarangzai, A. M.; Khan, N,; Wahab. M.; Kakar, A., 2010: New spread of dwarf mistletoe (Arceuthobium oxycedri) in Juniper forest, Ziarat, Balochistan, Pakistan. Pak. J. Bot. 42, 3709-3714.

Shaw, D.; Chen, J.; Freeman, E.; Braun, D., 2005: Spatial and population characteristics of dwarf mistletoe infected trees in an old-growth Douglas-fir - western hemlock forest. Can. ]. For. Res. 35, 990-1001.

Stoyan, D; Penttinen, A., 2000: Recent applications of point process methods in forestry statistics. Stat. Sci. 15, 61-78.

Stoyan, D.; Stoyan, H., 1996: Estimating pair correlation functions of planar cluster processes. Biom. J. 38, 259-271.

Turrill, W. B., 1920: Arceuthobium oxycedri and its distribution. Bull. Misc. Inform. (Royal Gardens, Kew) 8, 264-268.

Ward, M.; Paton, D. C., 2007: Predicting mistletoe seed shadow and patterns of seed rain from movements of the mistletoe bird. Dicaeum hirundinaceum. Austral Ecol. 32, 113-121.

Watson, D. M., 2009: Determinants of parasitic plant distribution: the role of host quality. Botany 87, 16-21.

Watson, D. M., 2011: Mistletoes of southern Australia. Collingwood: CSIRO Publishing.

Watson, D. M.; Rawsthorne, J., 2013: Mistletoe specialist frugivores: latterday 'Jolnnny Appleseeds' or self-serving market gardeners? Oecologia 172, 925-932.

Watson, D. M.; Roshier, D. A.; Wiegand, T, 2007: Spatial ecology of a root parasite from pattern to process. Austral Ecol, 32, $359-369$.

Wenny, D. G., 2001: Advantages of seed dispersal: a re-evaluation of directed dispersal. Evol. Ecol. Res. 3, 51-74.

Wiegand, T.; Moloney, K., 2004: Rings, circles and null-models for point pattern analysis in ecology. Oikos 104, $209-229$.

Wiegand, T.; Gunatilleke, S.; Gunatilleke, N., 2007: Species associations in a hetrogeneous Sri Lankan Dipterocarp forest. Am. Nat. 170. E77-E95.

Wiegand, T.; He, F.; Hubbell, S. P., 2013: A Systematic comparison of summary characteristics for quantifying point patterns in ecology. Ecography 36, 092-103.

Wiegnad, T.; Moloney, K., 2014: Handbook of spatial point-pattern analysis in ecology. Boca Raton: CRC Press, Taylor and Francis Group.

Zavala, l.; Zavala, M., 1993: Global Positioning System as a tool for ecosystem studies at the landscape level: an application in the Spanish Mediterranean. Landsc. Urban Plan. 24, 95-104.

Zilka, P. J.; Tinnin, R. O., 1976: Potential avian influence in the distribution of dwarf mistletoe. Nortlwwest Sci. 50, 8-16. 